\title{
Reducing data acquisition for light-sheet microscopy by extrapolation between imaged planes
}

\author{
Ziv Shemesh ${ }^{1} \mid$ Gal Chaimovich ${ }^{1} \mid$ Liron Gino $^{1} \mid$ Nisan Ozana $^{1} \mid$ Jonathan Nylk $^{2} \mid$ Kishan Dholakia $^{2} \mid$ Zeev Zalevsky*1
}

1 Faculty of Engineering and the Nanotechnology center, Bar Ilan University, Ramat-Gan, 52900, Israel

2 SUPA, School of Physics \& Astronomy, Physical Science Building, North Haugh, St Andrews, KY16 9SS, United Kingdom

\section{* Correspondence}

Zeev Zalevsky, Faculty of Engineering, Bar Ilan University ,52900, Ramat Gan, Israel

Email: zeev.zalevsky@biu.ac.il

\begin{abstract}
Light sheet fluorescence microscopy (LSFM) is a powerful technique that can provide high resolution images of biological samples. Therefore, this technique offers significant improvement for 3D imaging of living cells. However, producing highresolution $3 \mathrm{D}$ images of a single cell or biological tissues, normally requires high acquisition rate of focal planes, which means a large amount of sample sections. Consequently, it consumes a vast amount of processing time and memory, especially when studying real-time processes inside living cells. We describe an approach to minimize data acquisition by interpolation between planes using a phase retrieval algorithm. We demonstrate this approach on LSFM datasets and show reconstruction of intermediate sections of the sparse samples. Since this method diminishes the required amount of acquisition focal planes, it also reduces acquisition time of samples as well. Our suggested method has proven to reconstruct unacquired intermediate planes from diluted datasets up to $10 \mathrm{x}$ fold. The reconstructed planes were found correlated to the original pre-acquired samples (control group) with correlation coefficient of up to $90 \%$. Given the findings, this procedure appears to be a powerful method for inquiring and analyzing biological samples.
\end{abstract}

\section{KEYWORDS}

Light sheet microscopy, super resolution, Gerchberg-Saxton algorithm

\section{1 | INTRODUCTION}

A primary goal in biomedical studies is to map the real-time processes that occur in cells [1]. In order to conduct quantitative experiments in the field of life sciences, it is necessary to observe biological processes in vivo in real time. It is essential to achieve high axial resolution and high spatial resolution without compromising the physiological development of the specimen over a long period of time [2]. Generating a 3D model of the specimens, usually relies on $\mathrm{Z}$ stacks - a digital process of merging optical sectioning images to a $3 \mathrm{D}$ model, which is the basic technique of visualizing $3 \mathrm{D}$ structures [3].

There are several techniques for producing optical sectioning. All techniques that use imaging by sections refer terminologically to tomography. The method of tomography was first developed in the 1930 s by the radiologist Alessandro Vallebona. Focal plane tomography is based on the fact that the specimen appears sharper at the focal plane, while other planes appear blurred. This enables one to focus on each section of the specimen at a time and acquire each focal plane individually to eventually construct a 3D structure of the sample. Over the years, this method was developed, improved and branched out to various types and techniques in order to maximize efficiency and overcome limitations in different fields of study.

Bright-field light microscopy is one of the simplest and fundamental techniques for optical sectioning [4-5]. The principle action of this technique is based on the physical concept of absorption that leads to attenuation of light. The sample is illuminated from below, causing contrast changes due to different density areas in the sample. Observed from above, the sample typically seen as dark on bright background, hence the technique name. Unfortunately, this technique suffers from low contrast, low resolution and difficulty to observe colorless and transparent samples. The last become crucial when the study is focused on mammalians cells, as they are mainly transparent. 
Fluorescence microscopy is used as an imaging method that overcomes bright-field microscopy limitations [6]. In fluorescence microscopy the sample is illuminated with specific wavelengths which are absorbed by its fluorophores and emitted at different wavelengths. This method allows the sectioning to be more specific and therefore enhances the resolution of the imaged sample. The ability to image transparent specimens with improved resolution makes it widely used in biology, e.g., in living cells and biological tissues. On the other hand, illuminated samples are prone to photobleaching. The process of photobleaching occurs as the fluorescence molecules accumulate chemical damage from the excited electrons during fluorescence. As a result, the fluorophores lose their ability to fluoresce which can severely limit the observation time. Moreover, the method limits observations to specific structures which have been labelled for fluorescence.

Another well-known imaging technique for increasing optical resolution and contrast is called confocal microscopy, a special type of fluorescence microscopy [6-9]. Confocal microscopy uses two pinhole apertures positioned at confocal positions to block out-of-focus light in image formation. Since the light beam is focused only on a small part, the surface of the sample is scanned by moving either the sample or the light beam to allow plane reconstruction at specific height with a good spatial resolution. However, this can be very lengthy and tiresome when used for imaging a large amount of 3D structures data and also causes undesired effects such as photo-bleaching and photo-toxicity to the imaged planes.

Recent studied also shown the use of imaging by holography [10-12]. Holography uses diffraction to reproduce 3D light field, resulting an image to retains its depth, parallax and other original properties of the observed sample to achieve an accurate image reproduction. The technique enables a light field to be recorded and later reconstructed when the original light field is no longer in present, due to the absence of the original object. In common arrangement of this technique, the illuminating laser beam is split into two, one is projecting the sample and the other is used as a reference beam. The reflected and scattered light from the object is collected by the recording medium. The reference beam is expanded and shines directly on the recording medium, interacting with the light coming from the sample to create desired interference pattern. Nevertheless, this method has several drawbacks like being time consuming, high cost, may also suffer from noise and sensitive issues.

In order to overcome these limitations, light sheet-based fluorescence microscopy (LSFM) has recently emerged as an attractive imaging solution for biological specimens [13]. This method overcomes the disadvantages of the more conventional point scanning confocal or multiphoton methods mentioned above due to its orthogonal position between the illumination and detection arms. This reduces photodamage and allows fast acquisition making LSFM a preferred technique for imaging biomedical samples in neuroscience and developmental biology [14].

Note that LFSM technique is very useful for deep imaging within whole organisms or within transparent tissues. The tissues are exposed to only a thin plane of light, and thus there is less effects of photo-bleaching and photo toxicity caused to the specimen, compared to other methods as wide field fluorescence, confocal, or multiphoton microscopy [15]. Moreover, its depth of field (DOF) is lower and produces a higher-resolution image along the axial dimension [16]. The photo-toxicity bleaching is highly reduced due to a single interest plane illumination at a time [16-20].

In order to achieve high $3 \mathrm{D}$ resolution of structure by using a Z-stack in LFSM, a large number of image planes are required, i.e., the distance between the serial planes needs to be small, such that the 3D object is appropriately sampled. This requires a significant investment in both time and memory consumption.

In this paper, we present a modified Gerchberg-Saxton (GS) phase retrieval algorithm, in order to minimize the number of images per volume that are required for reconstructing a $3 \mathrm{D}$ image with sufficient axial resolution. In this way, we can retrieve the phase of the electro-magnetic field (EMF) and free space propagate the field in order extrapolate the axial information between the sampled reference planes. As we demonstrate via experimental results, the reconstructed intermediate planes have high correlation to the original images and therefore the reconstruction obtained with the proposed method is reliable and could be made applicable in light sheet microscopy. This method enables one to use a tenfold reduced axial sampling density in the imaging process, yet still retains the same information content while causing lower photo-damage, utilizing lower amount of memory, and exploiting higher speed of data acquisition.

\section{2 | THEORETICAL BACKGROUND}

\section{1 | GS Phase retrieval algorithm}

The GS algorithm is an iterative algorithm that is used, inter alia, for phase retrieval. Consider two plane images with known intensities $\mathrm{I}_{1}, \mathrm{I}_{2}$ and their EMF amplitudes:

$$
A_{1}=\sqrt{I_{1}}, \quad A_{2}=\sqrt{I_{2}}
$$

with unknown phases $\varphi_{1}, \varphi_{2}$ such that the EMF is:

$$
E_{1}=A_{1} \exp \left(i \varphi_{1}\right), \quad E_{2}=A_{2} \exp \left(i \varphi_{2}\right)
$$

with known distance between the planes denoted as $\Delta \mathrm{z}_{12}$ [21] The input amplitude $A_{1}$ is imposed with an initial random phase $\varphi_{1}$. Then, $E_{1}$ is propagated by free space propagation (FSP) distance of $z_{2}$ to obtain:

$$
E_{2}=F S P\left(E_{1}, \Delta z_{12}\right)=\widetilde{A_{2}} \exp \left(i \arg \left(E_{2}\right)\right)
$$

The argument of $E_{2}$ is kept while the amplitude of $\widetilde{A_{2}}$ is omitted and replaced by the known amplitude $A_{2}$.

$$
E_{2}=A_{2} \exp \left(i \arg \left(E_{2}\right)\right)
$$

Then, in the same manner, the reversed process is executed to obtain $E_{1}$ from $E_{2}$. 


$$
E_{1}=F S P\left(E_{2},-\Delta z_{12}\right)=\widetilde{A_{1}} \exp \left(i \arg \left(E_{1}\right)\right)
$$

After imposing the amplitude, one obtains:

$$
E_{1}=A_{1} \exp \left(i \arg \left(E_{1}\right)\right)
$$

The algorithm, as described, is performed iteratively, until reaching a desired correlation threshold $\rho$. Chart flow of the algorithm is described in Figure 1.

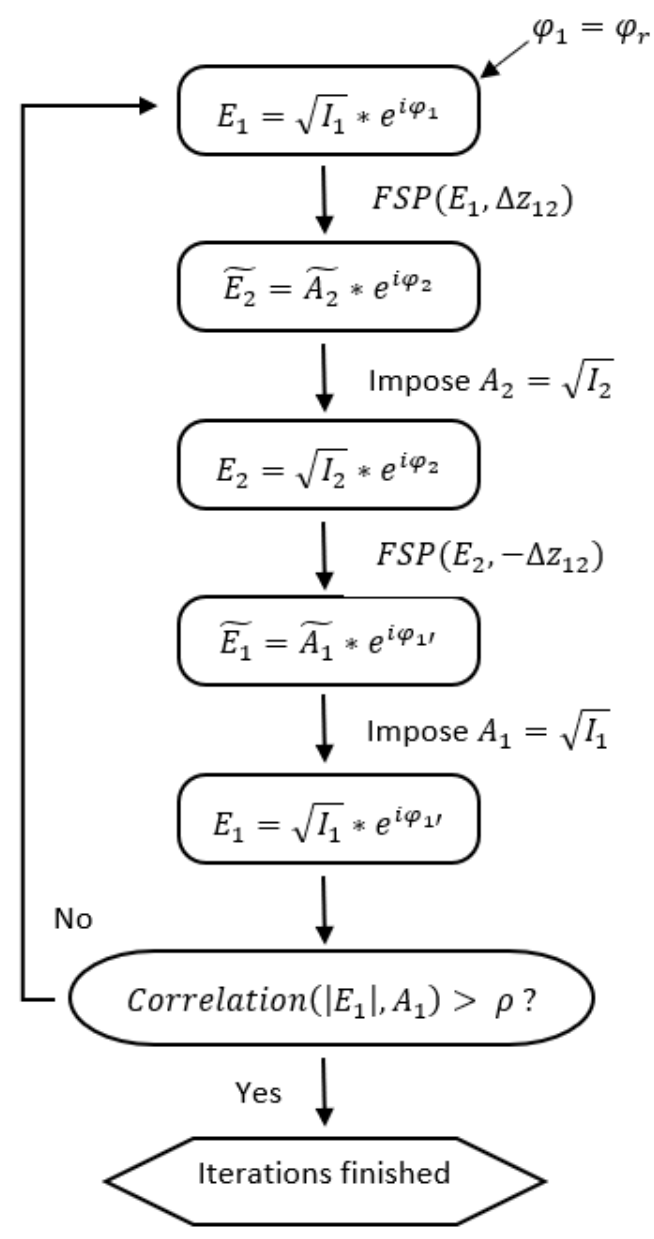

FIGURE 1 A flow chart describing the GS phase retrieval algorithm.

\section{$2.2 \mid$ LSFM}

As previously mentioned LSFM is a non-destructive imaging method for optical sectioning and visualizing tissue 3D, by using a plane (sheet) of light to reach a subcellular resolution (see Figure 2).
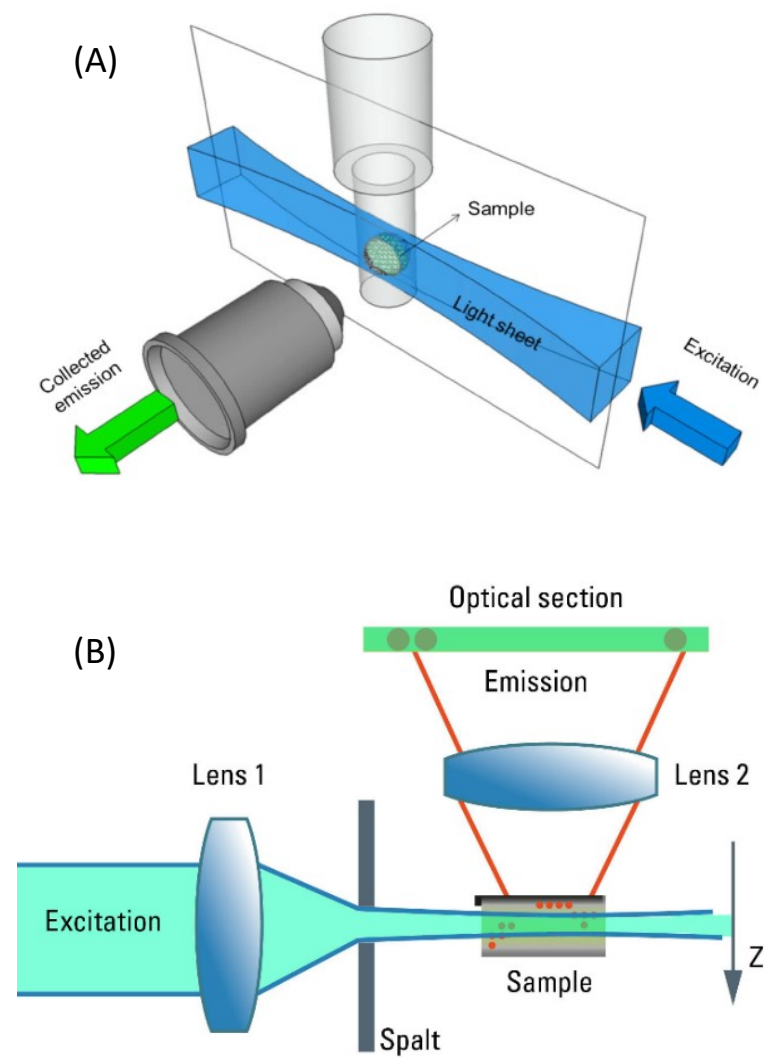

FIGURE 2 Schematic diagram of the light-sheet fluorescence microscopy of different sample types. (A) Spheroid [22]. (B) Beads [23].

\section{3 | MATERIALS AND METHODS}

\section{1 | Light-sheet data sets}

In this paper, we applied the modified algorithm on microscopic cellular datasets acquired by two-photon lightsheet fluorescence microscopy [14]. For the sectioning, a digitally scanned light-sheet fluorescence microscope (DSLM) [1], based on the openSPIM geometry [24], was assigned. The first data set contains volumetric image stacks of a 3D cultured cellular spheroid with dimeter of $\sim 450 \mu \mathrm{m}$. The Human Embryonic Kidney cells (HEK 293 T17) were plated in an ultra-low attachment 96-well round bottom cell capture plate (Corning ${ }^{\circledR}$ Costar ${ }^{\circledR}$ 7007) and grown for 48 hours. After the spheroids were formed, PUREBLUTM Hoechst 33342 nuclear staining dye was used to label their outer layer. The spheroids were embedded in $1 \%$ agarose in a FEP (Fluorinated Ethylene Propylene) capillary. The second data set includes image stacks of the sub-diffraction limited fluorescent bead. The $1 \mu \mathrm{m}$ diameter blue fluorescing beads were embedded in $1.5 \%$ agarose in a FEP capillary. The lightsheet system used in 2-photon excitation mode was obtained with an excitation central wavelength of $800 \mathrm{~nm}$. The laser power was approximate $50 \mathrm{~mW}$ and the camera exposure time was $100 \mathrm{~ms}$ in all the data sets. The NA of the lightsheet illumination objective was about 0.17 which corresponds to a light-sheet thickness (and axial resolution) of about $1.5-1.7 \mu \mathrm{m}$. The axial resolution will be best in the 
center of the image and increase to about $2.5 \mu \mathrm{m}$ after moving about $40 \mu \mathrm{m}$ away from the center (to the left or right). The NA of the detection lens is 0.5 which should give a lateral resolution of about $0.5 \mathrm{um}$. The camera has pixels of $6.5 \mathrm{um}$, and the system has a magnification of $40 \mathrm{x}$, therefore, each pixel is $0.1625 \mu \mathrm{m}$. The $\mathrm{z}$-spacing between each image plane is $0.25 \mu \mathrm{m}$.

\subsection{Correlation between source images}

For each data set, we tested the correlation between adjacent source images to examine the rate of changes and determine whether it is adequate for phase retrieval. Later, in order to determine an appropriate number of base planes to be used by the modified algorithm, in each dataset, we examined the correlation value between plane-images with difference of 5 , 10 and 20 planes between images of the same dataset. Meaning that the examined plane numbers were 1,11,21, etc. in each data set. Figures 3 and 4 show the correlation graphs between sequential images at different increments within the spheroid and the beads data sets respectively.

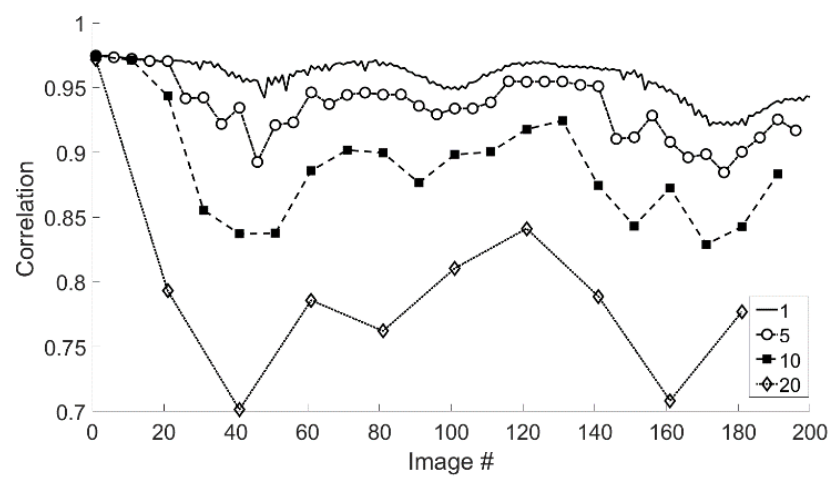

FIGURE 3 Correlation between the spheroid source images in planes separation of 1, 5, 10, 20 increments.

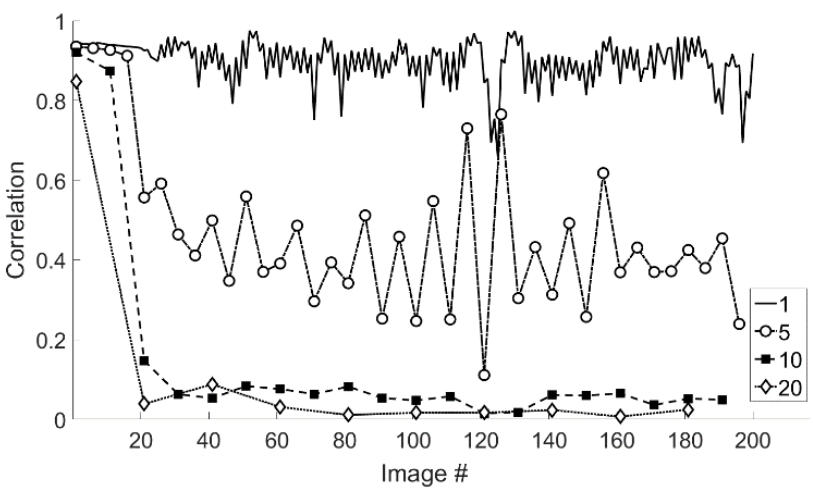

FIGURE 4 Correlation between the beads source images in planes separation of $1,5,10,20$ increments.
As seen in Figure 3, the correlation value between successive images is equal to or exceeds 0.95 along the entire dataset and remains above 0.82 up to increments of plane separation of 10. This indicates that relatively there are no rapid changes along the dataset, and we can choose base-plane images for the modified algorithm respectively.

For the dataset of the beads (see Figure 4), the correlation between successive plane-images remains high and around 0.9 , whereas when using increments by 5 , a sharp decrease was observed after few planes that worsen when using increments of 10 and 20 .

\section{3 $\mid$ Methods}

In the current approach, we have modified the known GS algorithm to set of multiple pre-known amplitudes Z-stack imaging data in order to diminish the number of z-plane images by reconstructing intermediate planes along the data set. For the proof of concept, five original base plane images have been taken with an increment of 10 images from one to the next (i.e., images number 1, 11, 21, 31 and 41). We have implemented a modified GS algorithm on the datasets in order to reconstruct the phase on each base plane image. In the same manner as shown above for the basic GS algorithm, the modified algorithm requires known amplitudes - taken from the datasets as our base plane images. These amplitudes we denoted as $A_{1}-A_{5}$ and they correspond to images 1,11 , 21,31 and 41 respectively. As in the basic algorithm, here as well we start with a random phase imposed to the initial amplitude $A_{1}$.

Now, instead of propagating the EM field between 2 planes $A_{1}$ and $A_{2}$, the modified algorithm executes propagation between all 5 known amplitudes $A_{1}-A_{5}$. After imposing the random phase for $A_{1}$ to create $E_{1}$, the field is propagated by FSP to $E_{2}$ and imposed with the known amplitude $A_{2}$ (3), (4). This propagation is then implemented in the same way between the rest of our sequential base planes $A_{2}-A_{3}, A_{3}-$ $A_{4}$ and $A_{4}-A_{5}$. The same propagation is now performed backwards from $A_{5}$ to $A_{1}$, like shown in the basic algorithm for amplitudes $A_{1}$ and $A_{2}$ (5), (6) to complete 1 iteration. Each iteration is described as flow chart in Figure 5. 


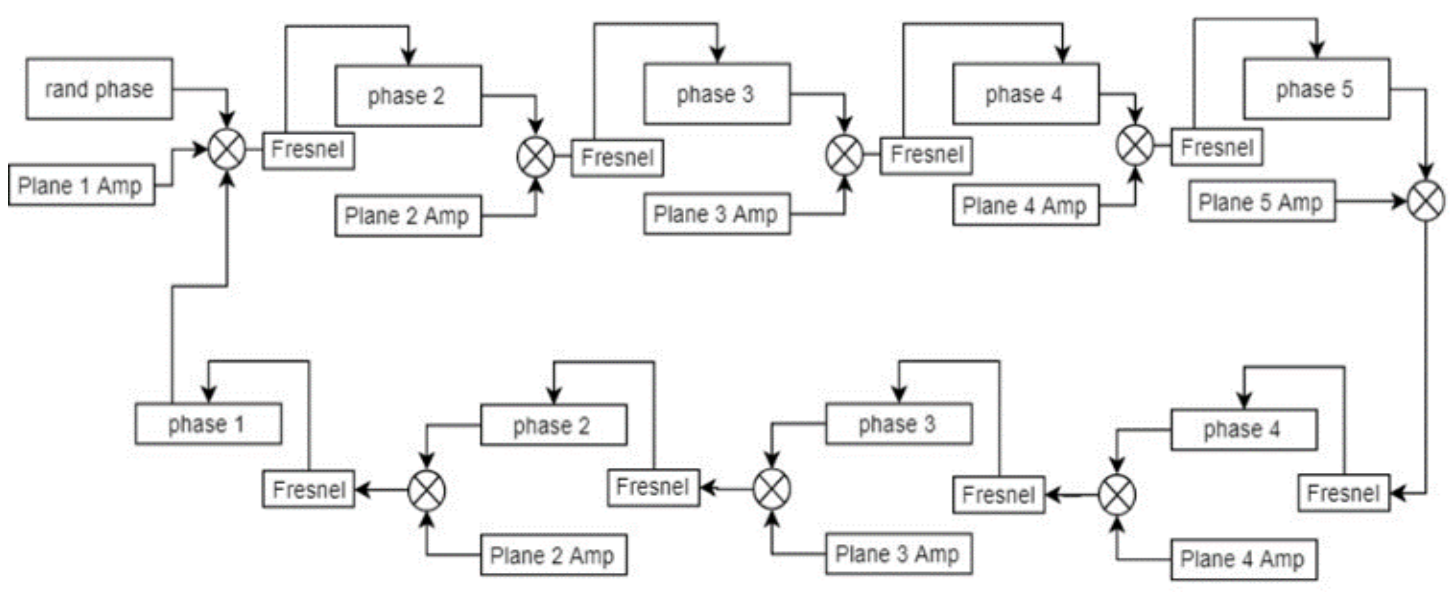

FIGURE 5 Modified GS algorithm's flow chart.

This modified algorithm has been applied using 100 iterations to achieve a single-phase reconstruction on each base plane. In that manner, the algorithm was executed 300 times with a different random phase at the entrance to obtain 300 reconstructed phases for every base plane (total of $100 \times 300$ iterations). At the end of the process, the reconstructed fields at the entrance plane (same amplitude with 300 different retrieved phases) was free space propagated to an intermediate plane (the plane of image 6 at $\mathrm{z}=1.25 \mu \mathrm{m}$, then averaged to a single image and compared to the original image of that plane. After implementing this algorithm, the resulted reconstructed images were analysed and compared to their original paralleled image at the same z-plane to measure and quantify the reconstruction quality. For the spheroid dataset we achieved correlation value of 0.97 and RMSE of 0.37 between the images, and for the beads set we obtained correlation of 0.9932 and RMSE of 0.25 . This means that the algorithm performed well and was able to reconstruct the original reference data.

Based on our empirical experiments and results, the recommended work point is 10 times lower z-sampling. Meaning that, in our case, the credible data is in resolution up to 1-2 micron. Although, light sheet microscopy technique can handle even lower dimensions, this is considered negligible compare to the benefits earned by using this method. An example of reconstruction is seen in Figure 6.

\section{4 | EXPERIMENTAL}

\subsection{Optimization}

For better accuracy and correlation improvement, the reconstructed planes were filtered with spatial Gaussian filter. This 2-D Gaussian shape filters an image with a smoothing kernel with standard deviation designated as $\sigma$ by performing convolution on the entire image. In this way, the side effect of added noise at the end of the iterations process can be diminished. In order to find the optimal standard deviation (STD) $\sigma$ of the Gaussian filter, we examined the Gaussian filter on the reconstructed image in plane 6. Different values of sigma between 0.5 to 100 , incremented by 0.5 , have been tested in order to find the optimal value of STD that yields the maximal correlation between the original and the reconstructed filtered image.
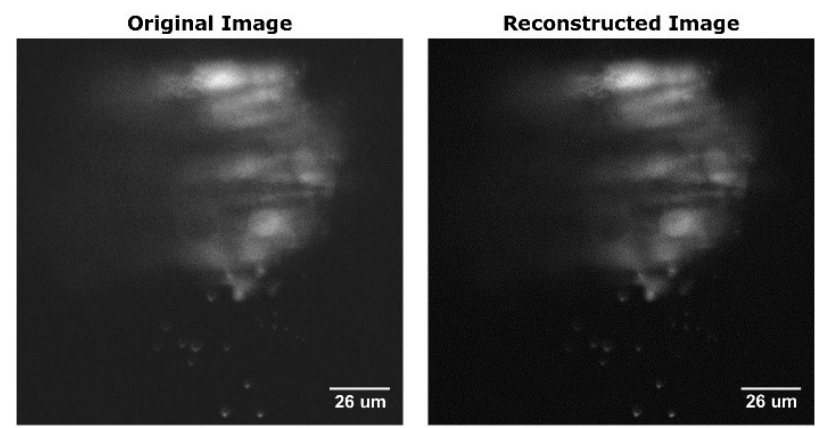

FIGURE 6 The reconstructed image 6 vs. original image taken from the spheroid dataset.

From the obtained results, we saw that for the spheroid data, filter with STD of 4 pixels gave the highest correlation and in the beads data a filter with STD of 2.5 pixels gave the highest correlation to the data set.

\subsection{Number of random phases dependency}

A further correlation test was applied to explore the dependency on the number of random phases. The experiment was performed and distinguished between results of before filtering and after filtering. This correlation test has shown with clarity that the correlation value before applying the Gaussian filter is better for a more significant number of random phases. However, it is significantly noticeable also that the improvement in the correlation after using the Gaussian filter is almost unchanged, independently on the number of random phases.

The correlation trend practically remained the same for 10 or even 300 random phases. These findings allow performing this modified GS algorithm with high efficiency and decent image reconstruction using only few base-planes source images. 


\section{5 | RESULTS AND DISCUSSION}

Following the obtained results from the experimental tests, the modified algorithm has been executed on the entire data sets. Base planes were taken from each data set in the same manner to perform the modified algorithm. 10 random phases have been retrieved in each base plane and reconstructed intermediate planes were filtered according to the suitable optimal Gaussian filter.

The obtained results showing the correlation between all reconstructed images vs. original images for the spheroid data set are seen in Figures 7 and 8.

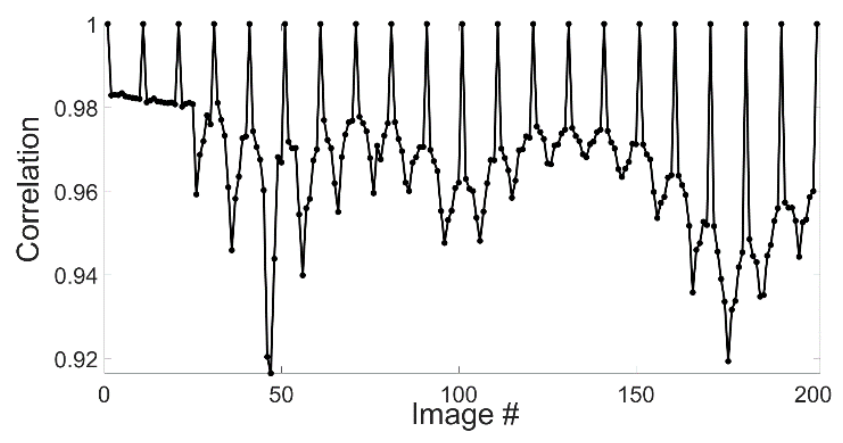

FIGURE 7 The correlation obtained between all reconstructed images vs. original images for spheroid dataset.

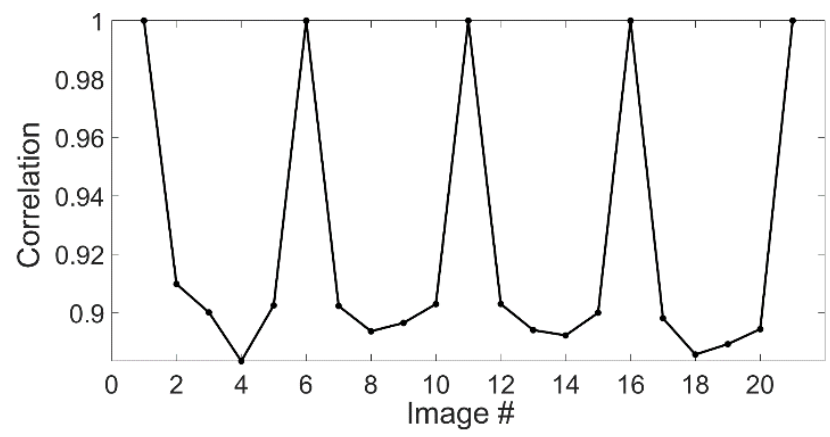

FIGURE 8 The correlation obtained between the first 21 reconstructed images vs. original images for beads dataset.

One may see that after applying the Gaussian filter correlation value above 0.93 was obtained across the entire data set. which indicates that good reconstruction was obtained. An example of original, reconstructed and reconstructed-filtered image can be seen in Figure 9 and 10. In addition, a 3D rendering of the spheroid data can be observed in respect to $\mathrm{x}-\mathrm{z}$ plane as shown in Figure 11 (orthogonal to Figure 9 that shows $\mathrm{x}-\mathrm{y}$ plane observation of the spheroid.

We demonstrated an application of our modified algorithm on sparse datasets of spheroid and fluorescent beads. Though the results proven applicable on our given datasets, it should be taken under consideration that dilution factor may vary between different types and size of interested objects. For instance, we have showed in Figures 3 and 4, correlation plots of our datasets, applying different dilution factors. As mentioned, and based on the results, we therefore determined different dilution factor for each dataset.

In our case, the demonstrated dilution factors varied between a factor of 5 and 10 . In case the data is not sparse but rather contains several different types of objects within the field of view, then our approach will include a preliminary step of classification and segmentation and then the iterative algorithm will be applied separately on each segmented region in the field of view that correspond to different type of objects. In this way the presented improvement factor of 5-10 can still be preserved even in more dense images containing several types of objects per frame.

For example, if the object of interest consists of minute corpuscles, the algorithm dilution factor could be modified, localized and implanted in segmentation for different areas within the sample. Dilution factor should be determined by the sample size, rate and correlation between sequential planes of the diluted data.

\section{4 | CONCLUSION}

This paper demonstrates a technique for 3D imaging of microscopic cellular data sets acquired by two-photon lightsheet fluorescence microscopy, using 10 times smaller number of plane-images than would typically be required. Thus, it is more suitable for live cell imaging, since it is less time consuming and therefore further reduce phototoxicity to the cell.

On the other hand, it should be acknowledged that there is still a minuscule amount of information loss. Moreover, the resolution of the sample decreased slightly due to reduction in the sampling rate. Consequently, it may be difficult to recognize organelles that are much smaller than the new lower sampling rate. 

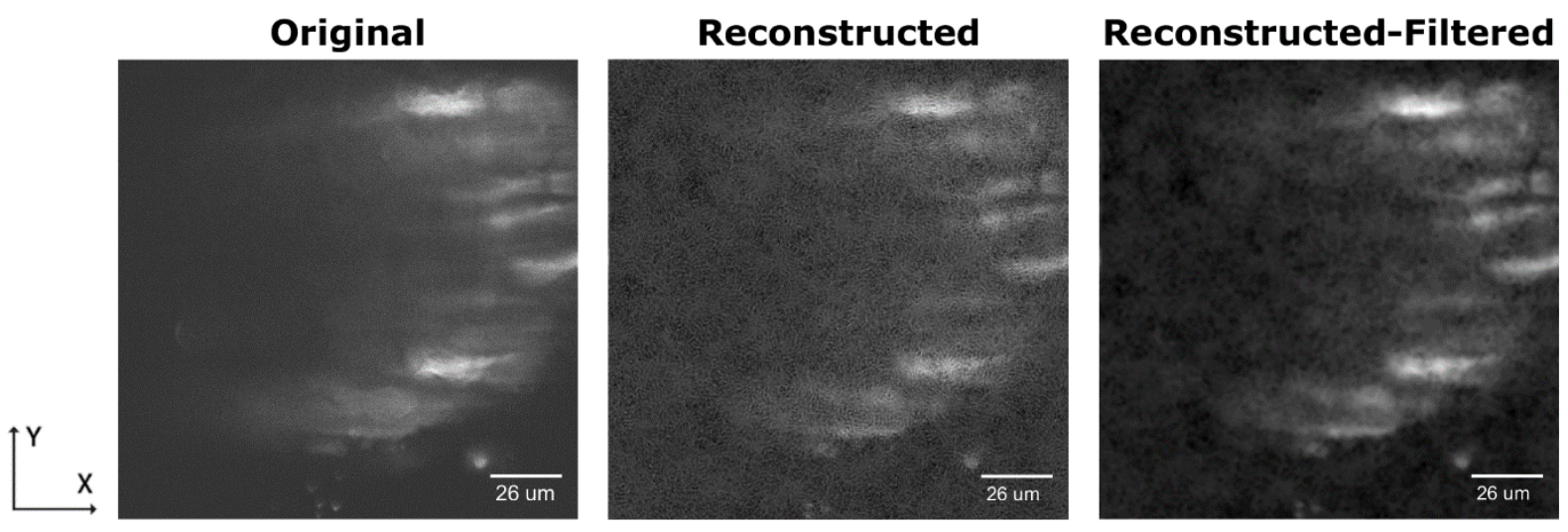

FIGURE 9 Original image number 106 from spheroid data set compared to reconstructed image and reconstructed-filtered image.
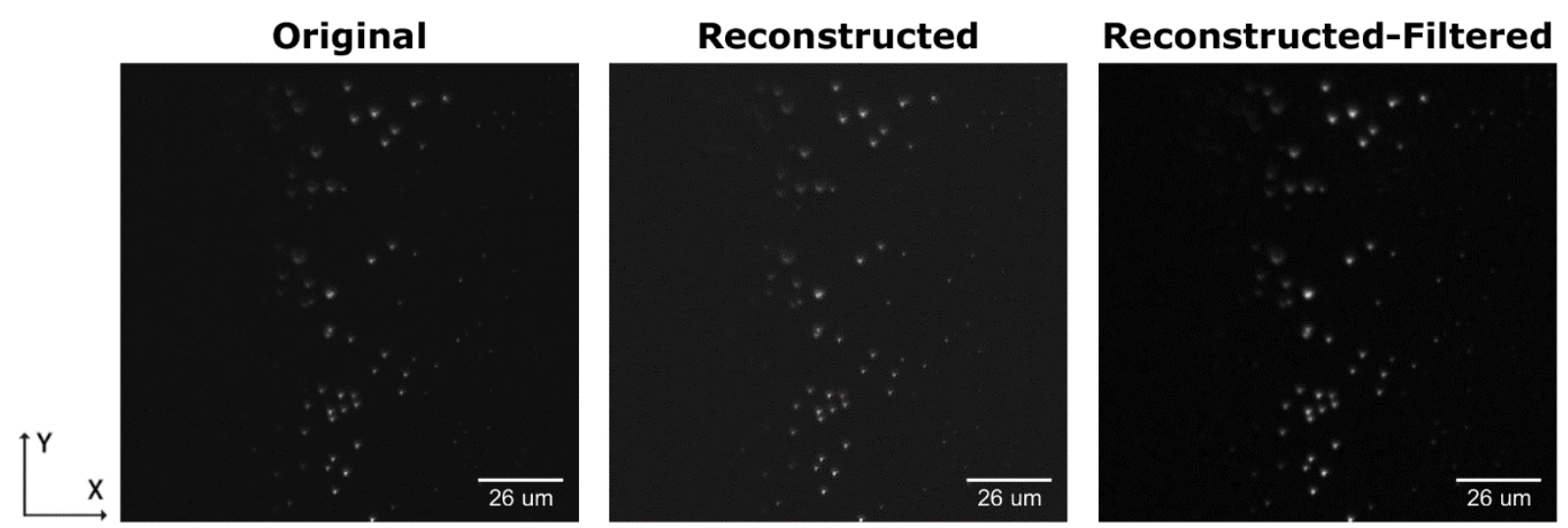

FIGURE 10 Original image number 13 from beads data set compared to reconstructed-filtered image.
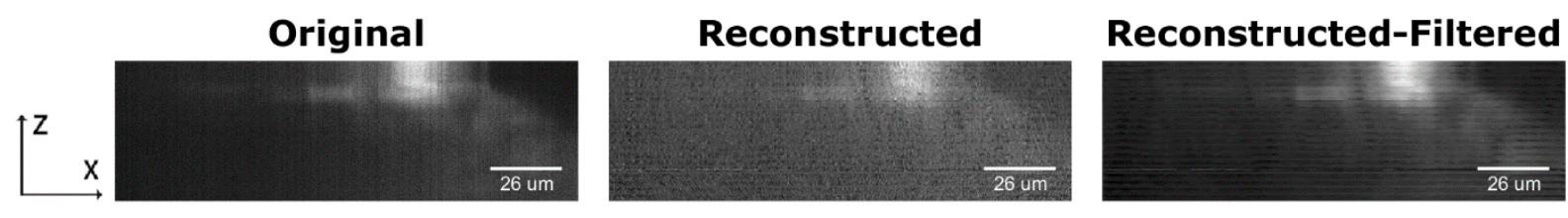

FIGURE 11 Original image number 106 from spheroid data set compared to reconstructed image and reconstructedfiltered image in respect to $\mathrm{x}-\mathrm{z}$ plane section.

\section{CONFLICT OF INTEREST}

The authors declare no conflicts of interest.

\section{REFERENCES}

[1] P. J. Keller, A. D. Schmidt, J. Wittbrodt and E. H.K. Stelzer, Science. 20083221065

[2] P. J. Keller and E. H.K. Stelzer, Current Opinion in Neurobiology. 200818624

[3] D. A. Agard, Annual Review of Biophysics and Bioengineering. 198413191

[4] D. Karadaglic, Micron, 200839302
[5] X. Ma, Z. Zhang, M. Yao, J. Peng and J. Zhong, Ultramicroscopy, 201819574

[6] E Fernandez, S. Marull-Tufeu, Skin Research and Technology 201925735

[7] T. Wilson and C. Sheppard, Theory and Practice of Scanning Optical Microscopy, Academic Press, London, 1984

[8] J. B. Pawley, Handbook of Biological Confocal Microscopy, Springer Science \& Business Media, New York, 2010

[9] M. Rajadhyaksha, M. Grossman, D. Esterowitz, R. H. Webb and R. R. Anderson, Jornal of Investigative Dermatology. 1995, 104 946

[10] F. Merola et al., Light: Science \& Applications, 2017, 6 e16241

[11] S. Shao, K. Mallery, S.S. Kumar ans J. Hong, Optics Express, 2020, 282987

[12] M. Ziemczonok, A. Kuś, P. Wasylczyk and M. Kujawińska, Scientific Reports. 201991 
[13] A. Maizel, D. von Wangenheim, F. Federici, J. Haseloff and E. H.K. Stelzer, The Plant Journal. 201168377

[14] A. Escobet-Montalbán, F. M. Gasparoli, J. Nylk, P. Liu, Z. Yang and K. Dholakia, Optics Letters. 2018435484

[15] P. A. Santi, Journal of Histochemistry \& Cytochemistry. 201159 129

[16] J. Huisken, J. Swoger, F. Del Bene, J. Wittbrodt and E. H.K. Stelzer, Science. 20043051007

[17] E. G. Reynaud, U. Kržič, K. Greger and E. H.K. Stelzer, HFSP Journal. 20082266

[18] E. H.K. Stelzer, Mechanisms of Development. 2009126 S36

[19] F. Pampaloni, N. Ansari, P. Girard and E. H.K. Stelzer, Advanced Microscopy Techniques II. $2011808680860 \mathrm{Y}$

[20] E. G. Reynaud, J. Peychl, J. Huisken and P. Tomancak, Nature Methods. 20141230

[21] T. Ilovitsh, A. Ilovitsh, A. Weiss, R. Meir and Z. Zalevsky, Scientific Reports. 2015515473

[22] O. E. Olarte, J. Andilla, E. J. Gualda and P. Loza-Alvarez, Advances in Optics and Photonics. 201810111

[23] E. J. Gualda, H. Pereira, G. G. Martins, R. Gardner and N. Moreno, Cytometry Part A. 201791144

[24] P. P. Pitrone, J. Schindelin, L. Stuyvenberg, S. Preibisch, M Weber, K. W. Eliceiri, J. Huisken and P. Tomancak, Nature Methods. 2013 